\title{
A modification of the TIP procedure for distal hypospadias: The burrowing technique
}

\author{
Erin D. Wishart, MD; Peter D. Metcalfe, MD
}

Department of Surgery, Division of Urology, University of Alberta, Edmonton, AB

Cite as: Can Urol Assoc J 2014;8(5-6):e425-8. http://dx.doi.org/10.5489/cuaj.1677 Published online June 19, 2014.

\section{Abstract}

Introduction: The tubularized incised plate urethroplasty (TIP) hypospadias repair is a commonly performed procedure for hypospadias. Multiple series document excellent cosmetic outcome in conjunction with low complication rates. We describe a modification that we have named the "burrowing technique." We believe that this technique facilitates dissection of the glans, which improves mobility, decreases tension with closure, and potentially improves outcomes.

Methods: A retrospective review was performed of 193 coronal or mid-shaft hypospadias repairs by a single surgeon. The first 98 were performed using the TIP procedure, then the burrowing technique was developed and a subsequent 95 were analyzed for outcomes using this modification. Urethral plate characteristics and glandular size did not influence the choice of surgical technique. Cases were selected to allow for a "learning curve," and were consecutively accrued. None of the boys had undergone prior hypospadias surgery. Proximal 2 stage repairs and distal (glanular) repairs were excluded.

Results: A total of 193 boys underwent repair, 98 with the traditional TIP procedure and 95 using the burrowing modification. In total, $37(19.2 \%)$ patients required re-operation for either fistulas or dehiscence; 23 (23.5\%) in the non-burrowing group and 14 $(14.7 \%)$ in the burrowing group, odds ratio $0.54(p=0.10)$.

Conclusions: The TIP procedure has revolutionized the management of distal hypospadias. The burrowing modification increases glandular mobility simplifying the procedure and demonstrates a non-statistically significant trend in reducing reoperation rates.

\section{Introduction}

Hypospadias is one of the most frequently observed birth anomalies of the urogenital system, with an incidence of about $0.2 \%$ to $0.4 \%{ }^{1} \mathrm{~A}$ large proportion of these cases are distally located. ${ }^{2}$ Despite this, there is no consensus on the optimal surgical technique for distal hypospadias. ${ }^{3,4}$ There are over 300 surgical techniques described for repairing hypospadias. ${ }^{2,5}$ However, the Snodgrass tubularized incised plate urethroplasty (TIP) is perhaps the most commonly performed contemporary repair. ${ }^{6}$ A study done by Springer and colleagues surveyed surgeons who operate on hyposadias and found that in distal hypospadias (subcoronal to midshaft), the TIP repair was preferred by $52.9 \%$ to $71.0 \%$ of participants. ${ }^{3}$ Nevertheless, relatively high rates of meatal stenosis and/or urethrocutaneous fistula (up to 33\%) have been a concern with this procedure. ${ }^{7,8}$ Creating a tensionfree repair by increasing the mobility of the neourethra and glans is an important factor in determining the success of the surgery and in preventing complications.

Over the past 4 years, we have been using a modification of the TIP procedure, named the "burrowing technique," that we believe facilitates the glanular dissection and results in increased mobility of the glans wings, potentially decreasing reoperation rates. Dissection of the divergent spongiosal tissue from the corpora facilitates the development of an anatomic plane, which can be followed up into the glans. This allows an easy and accurate dissection right to the tips of the corpora cavernosa and results in excellent glans mobility and preservation of anatomic blood supply.

In this study we compared the complication rates of distal hypospadias repair using the TIP procedure with the burrowing technique.

\section{Methods}

A retrospective review was preformed on a single surgeon's (PDM) coronal to mid-shaft hypospadias' cases over a 6-year period. These were consecutively accrued, excluded the surgeon's first 100 hypospadias repairs and allowed for a minimum 2-year follow-up. Re-do surgery and non-TIP repairs (glanular/urethral advancement repairs and proximal 2 stage repairs) were excluded. From October 2006 to October 2009, the TIP procedure was used. From October 2009 onwards, the burrowing technique was developed 
and used exclusively. Measurement of the glans size and the recording of the urethral plate characteristics were not routinely performed, as the surgeon did not use them to affect clinical decisions (i.e., the same technique was used regardless of glans and urethral plate size). Preoperative testosterone was not given to any patients.

The primary outcome was the need for a second surgery. This was further classified as: meatal stenosis, fistula, glanular dehiscence, or a combination of these complications. Age at the time of operation was also collected. Operation room reports and electronic medical records were reviewed to find which boys required reoperation for stenosis, fistula, or dehiscence based on the postoperative diagnosis at the time of the second surgery.

\section{Surgical technique}

\section{TIP}

Surgery is done under general anesthesia and prophylactic antibiotics are given. A traction suture is placed and the urethral plate is then incised, only through the epidermis. The penile skin is completely degloved and an artificial erection is performed. About 0.25 cc of $1 \%$ bupivacaine with $1 / 200000$ units of epinephrine is then injected into the glans. The glans is sharply dissected from the urethral plate with a scalpel, from the presumed external margin of the plate to the presumed appropriate depth. Urethra is closed in two layers using 7-0 PDS.

After the urethroplasty, a dartos flap is harvested and tacked in place using 7-0 PDS. The glans is closed over a 8-Ffr catheter using 6-0 PDS. Byers flaps are created, rotated ventrally and tailored to ensure adequate penile shaft coverage. The epidermis is closed using 6-0 vicryl. A 6-Fr urethral stent is sutured in place for 6 to 8 days and patients are given prophylactic septra or cephazolin for the duration of catheterization.

\section{Burrowing technique}

The technical details are identical to the TIP procedure - up until the degloving step. Once degloved, the burrowing technique starts by dissecting proximal to where the spongiosum diverges (Fig. 1). Here, a plane is developed between the coporal bodies and spongiosum. Only the lateral, divergent spongiosum is elevated, not the entire dorsal urethra, to preserve blood supply.

This plane is followed up to the glans. About $0.25 \mathrm{cc}$ of $1 \%$ bupivacaine with $1 / 200000$ units of epinephrine is injected along the developed plane to facilitate dissection. The distal margin of the urethra is assessed by determining the point of apposition around a 8-Fr catheter, and the urethral plate is outlined with a marking pen. Sharp tipped (tenotomy) scissors are used to develop the corporal plane by "burrowing" between the glans and corpora (Fig. 2). The urethra is then transected from the corpora by placing one blade of the scissors in the dissected plane and the second along the marked out urethral plate (Fig. 3). Dissection is deemed complete when the tips of the corpora are seen.

Urethroplasty, dartos flap, and glans closure are then performed in the same way as with the TIP procedure. With the burrowing modification, the mobilized divergent spongiosal tissue provides an excellent second layer of tissue coverage (Fig. 4).

\section{Results}

In total, 193 patients who underwent a primary coronal or subcoronal hypospadias repair were included in this study. Of these, 98 patients underwent the TIP procedure and 95 with the borrowing technique. There was no difference in the patients' ages (TIP mean $=19$ months [range: 6 months to 9 years]; burrowing mean $=17$ months [range: 8 months to 7 years).

Of the patients who underwent the TIP procedure, 23 $(23.5 \%)$ required additional surgery. In the burrowing cohort, 14 patients $(14.7 \%)$ required additional surgery (Table 1$)$. However, this did not reach statistical significance (odds ratio $0.54 \mathrm{Cl}$ [confidence interval] 0.26-1.13, $p=0.10$ ).

None of our patients had a meatal stenosis requiring surgical intervention. Of the 23 TIP revisions, 12 were due to a glandular dehiscence, 9 due to fistula, and 2 had both dehiscence and fistula. Of the 14 patients who required revisions with the burrowing technique, 8 reoperations were due to glandular dehiscence and 6 due to fistula formation. Use of the burrowing modification lowered the risk of dehiscence requiring a corrective surgery by $0.55(\mathrm{Cl}$ $0.22-1.38, p=0.20$ ). The risk of fistula requiring addition

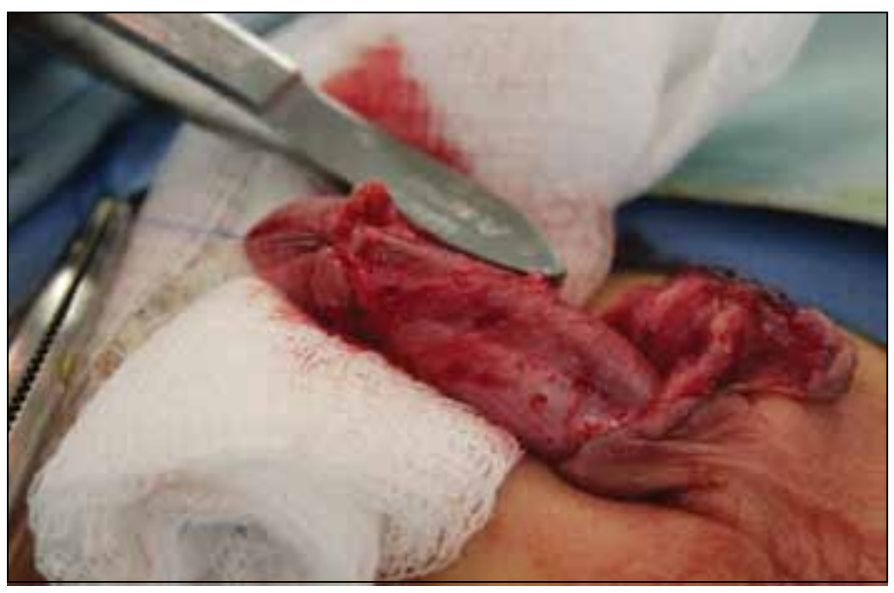

Fig. 1. The dissection is started proximal to where the spongiosum diverges from its normal tubularized form to the dysplastic, hypospadic portion. 


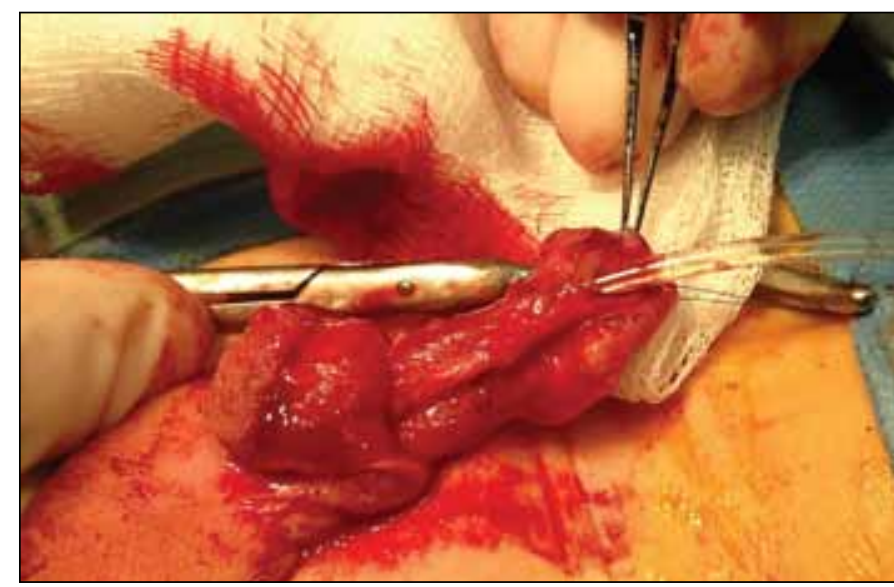

Fig. 2. The plane is developed along the corporal spongiosal border and followed up into the glans, between the urethral plate and glans by "burrowing" the scissors through the anatomic plane.

surgery was $0.53(\mathrm{Cl} 0.19-1.51, p=0.24)$ less likely using the TIP procedure compared to the burrowing modification (Table 2). However, these were not statistically significant.

\section{Discussion}

Elevation of the divergent spongiosum is an effective means of eliminating residual chordee, provides excellent tissue for the urethroplasty, and facilitates dissection along the corpora into the glans. Care must be taken not too devascularize the urethra to minimize risks of stenosis, as reported by Snodgrass and colleagues. ${ }^{9}$ Although the odds ratio was not statistically significant, the burrowing technique shows a trend towards decreasing the need for further surgery. Subjectively, the author prefers this modification as it seems more reliable, and it facilitates and expedites the glanular dissection. Finally, the improved glans mobility allows for a

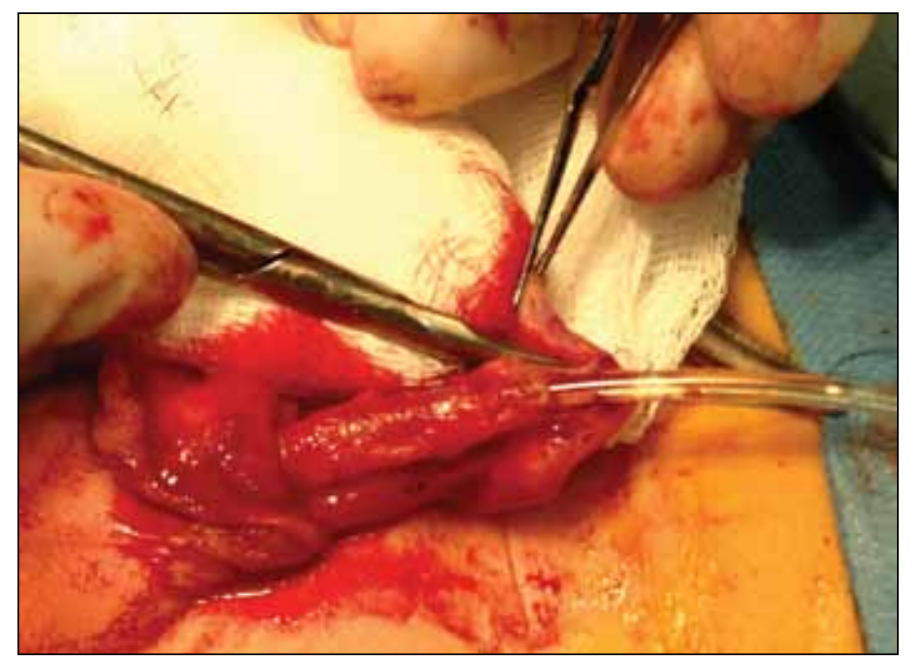

Fig. 3. Controlled incision is made through the previously developed plane. Angulation of scissors and the use of a marking pen will help control the amount of tissue used for the urethra and glans.

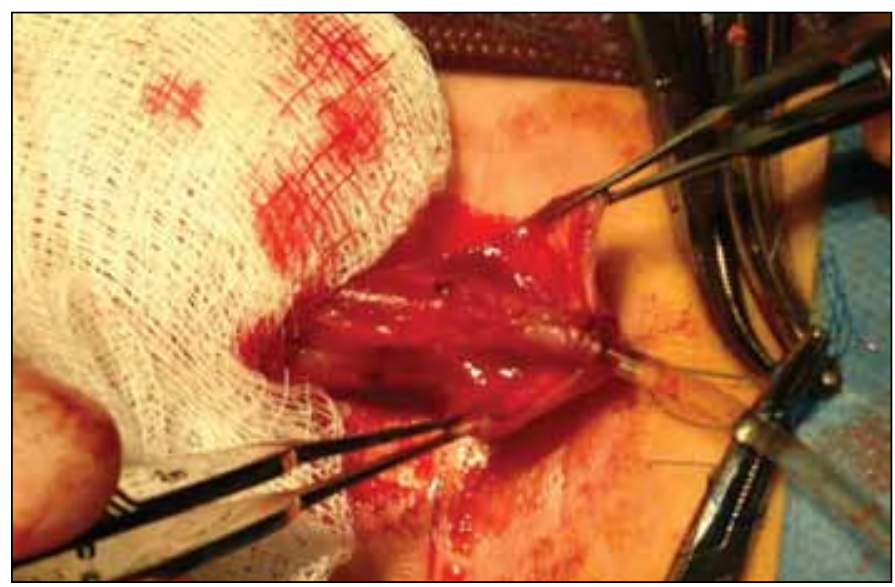

Fig. 4. Mobilized spongiosal tissure providing enhanced glandular mobility.

more conical and normal appearance to the glans (Fig. 5).

Despite its frequency, hypospadias surgery has a significant complication rate. ${ }^{8,10}$ Our revision rates are in keeping with published reviews, even with our exclusion of meatal and glanular lesions. Unfortunately, we were not able to demonstrate a decrease in the need for further surgery, but still feel that our modification has several benefits.

We attempted to minimize variability by excluding distal repairs (meatal and glanular hypospadias), as these patients would not benefit from improved glans mobilization. We

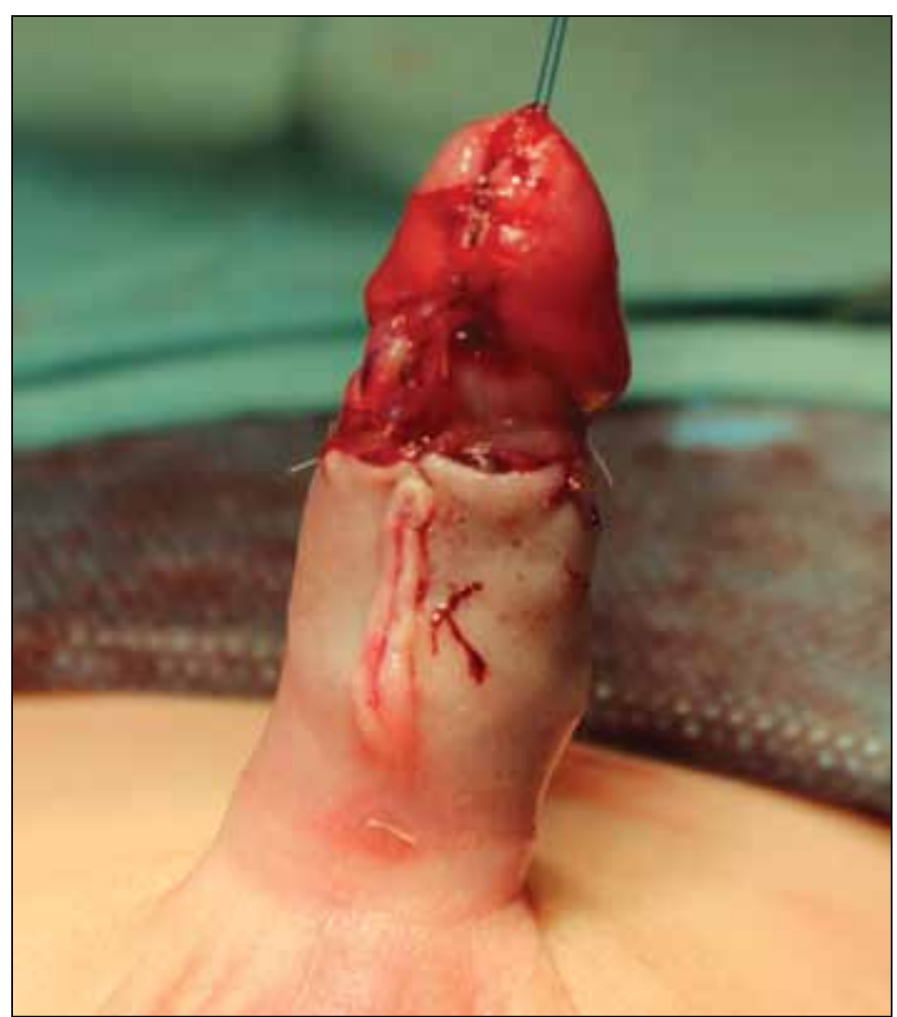

Fig. 5. Using the burrowing technique, we achieve a more conical and normal appearance to the glans. 


\begin{tabular}{|c|c|c|c|}
\hline & Reoperation & Total & \% Reoperation \\
\hline TIP procedure & 23 & 98 & 23.5 \\
\hline Burrowing technique & 14 & 95 & 14.7 \\
\hline Total & 37 & 193 & 19.2 \\
\hline
\end{tabular}

TIP: tubularized incised plate urethroplasty.

excluded the first 100 repairs over the first 2 years of practice, to allow for a "learning curve" as this may have also biased the results. We also allowed for a minimum of 2 years of follow-up. However, excluding these patients may have limited our total numbers, and affected the power of the statistical analysis.

Our definition of complications was a simple, but honest, representation. Obviously, some families may choose not to pursue further surgery after a complication, and this may have affected our results.

Furthermore, the study design was limited by the fact that glans size was not routinely measured, nor was the urethral plate characteristics routinely consulted. The authors do not measure or record urethral width or glans size, as the preoperative preparation or surgical technique does not vary accordingly. The ability to stratify based on glans size may have affected the study, as the smaller glans may have benefited more from the improved mobilization and decreased tension.

\section{Conclusion}

The tubularized incised plate urethroplasty has revolutionized the management of distal hypospadias. The burrowing modification described in this article may increase glandular wing mobility simplifying the procedure and showing a trend towards reducing the risk of reoperation.

Competing interests: Dr. Wishart and Dr. Metcalfe declare no competing financial or personal interests.

This paper has been peer-reviewed.

\begin{tabular}{lccc}
\hline \multicolumn{4}{l}{ Table 2. Type of complications } \\
\hline & TIP Procedure & Burrowing & Odds ratio \\
\hline Fistula & 11 & 6 & $0.53(p=0.24)$ \\
Dehiscence & 14 & 8 & $0.55(p=0.20)$ \\
\hline
\end{tabular}

TIP: tubularized incised plate urethroplasty.

\section{References}

1. Paulozzi U, Erickson JD, Jackson RJ. Hypospadias trends in two US surveillance systems. Pediatrics 1997;100:831-4. http://dx.doi.org/10.1542/peds.100.5.831

2. Subramaniam R, Spinoit A-F, Hoebeke P. Hypospadias repair: An overview of the actual techniques. Semin Plast Surg 2011;25:206-12. http://dx.doi.org/10.1055/s-0031-1281490

3. Springer A, Krois W, Horcher E. Trends in hypospadias surgery: Results of a worldwide survey. Eur Urol 2011;60:1184-9. http://dx.doi.org/10.1016/j.eururo.2011.08.031

4. Wilkinson DJ, Farrelly P, Kenny SE. Outcomes in distal hypospadias: A systematic review of the Mathieu and tubularized incised plate repairs. J Pediatr Urol 2012;8:307-12. http://dx.doi.org/10.1016/i. ipurol.2010.11.008

5. Macedo A, Rondon A, Ortiz V. Hypospadias. Curr Opin Urol 2012;22:447-52. http://dx.doi. org/10.1097/MOU.0b013e328357bc62

6. Steven L, Cherian A, Yankovic $F$, et al. Current practice in paediatric hypospadias surgery: A specialist survey. J Pediatr Urol 2013;9:1126-30. http://dx.doi.org/10.1016/i.jpurol.2013.04.008

7. Spinoit A-F, Poelaert F, Groen $L-A$, et al. Hypospadias repair at a tertiary care center: Long-term followup is mandatory to determine the real complication rate. J Urol 2013;189:2276-81. http://dx.doi. org/10.1016/i.juro.2012.12.100

8. Wang $F, X u Y$, Zhong $H$. Systematic review and meta-analysis of studies comparing the perimeatal-based flap and tubularized incised-plate techniques for primary hypospadias repair. Pediatr Surg Int 2013;29:81121. http://dx.doi.org/10.1007/s00383-013-3335-3

9. Snodgrass WT, Granberg C, Bush NC. Urethral strictures following urethral plate and proximal urethral elevation during proximal TIP hypospadias repair. J Pediatr Urol 2013;9:990-4.

10. Cimador M, Vallasciani S, Manzoni G, et al. Failed hypospadias in paediatric patients. Nat Rev Urol 2013;10:657-66.

Correspondence: Dr. Peter D. Metcalfe, Department of Surgery, Division of Urology, University of Alberta, Edmonton, AB; pmetcalf@ualberta.ca 